\title{
BEAM MEASUREMENTS AND UPGRADE AT BL 7.2, THE SECOND DIAGNOSTICS BEAMLINE OF THE ADVANCED LIGHT SOURCE*
}

\author{
Tom Scarvie, Fernando Sannibale, Alan Biocca, Nicholas Kelez, Michael C. Martin, Toshiro \\ Nishimura, Gregory Portmann, Eric Williams, LBNL, Berkeley, CA 94720, U.S.A.
}

\begin{abstract}
Beamline 7.2 of the Advanced Light Source (ALS) at the Lawrence Berkeley National Laboratory (LBNL) is a beam diagnostics system that uses the synchrotron radiation emitted by a dipole magnet. It consists of two branches; in the first one the $\mathrm{x}$-ray portion of the radiation is used in a pinhole camera system for measuring the transverse profile of the beam. The second branch is equipped with an x-ray beam position monitor (BPM) and with a multipurpose port where the visible and the farinfrared part of the radiation can be used for various applications such as bunch length measurements and IR coherent synchrotron radiation experiments. The pinhole system has been operating successfully since the end of 2003. The installation of the second branch has been completed recently and the results of its commissioning are presented in this paper together with examples of beam measurements performed at BL 7.2.
\end{abstract}

\section{INTRODUCTION}

BL 7.2, the second beam diagnostics dedicated beamline at the ALS $[1,2]$, is composed of two branches. The main purpose of the first branch is to measure the horizontal beam size at the second bending magnet of our triple bend achromat lattice. This measurement is then combined with an analogous measurement from BL 3.1, on the first bend magnet, to deduce the emittance and momentum spread of the electron beam $[1,3]$. This part of the beamline is basically a hard x-ray pinhole camera based on a design implemented earlier at BESSY II [4]. The second branch includes an x-ray BPM for orbit measurements, and a multipurpose port where the visible light is used for longitudinal beam distribution and dynamics measurements, and the far-infrared (FIR) portion of the synchrotron radiation used for coherent synchrotron radiation (CSR) experiments.

The installation of the pinhole part of the beamline was completed in August 2003, and the beamline is now in routine operation for transverse beamsize measurements, which are archived and trended for machine quality control. For a complete description of this part of the beamline and of its first measurements, see the commissioning paper from EPAC 2004 [2].

The second branch of the beamline, where an optical channel transports the visible light and the FIR synchrotron radiation outside the storage ring (SR), has

*Work supported by the Director, Office of Science, of the U.S.

Department of Energy under Contract No. DE-AC03-76SF00098. been instrumented and commissioned more recently.

In this paper we present a description of the recent upgrades of BL 7.2 and examples of the measurements performed in both the branches of the beamline.

\section{BL 7.2 FIR-VISIBLE BRANCH DESCRIPTION}

Figure 1 shows an engineering view of the beamline. The source point is located in the central bend of the triple bend achromat cell of Sector 7 of the ALS.

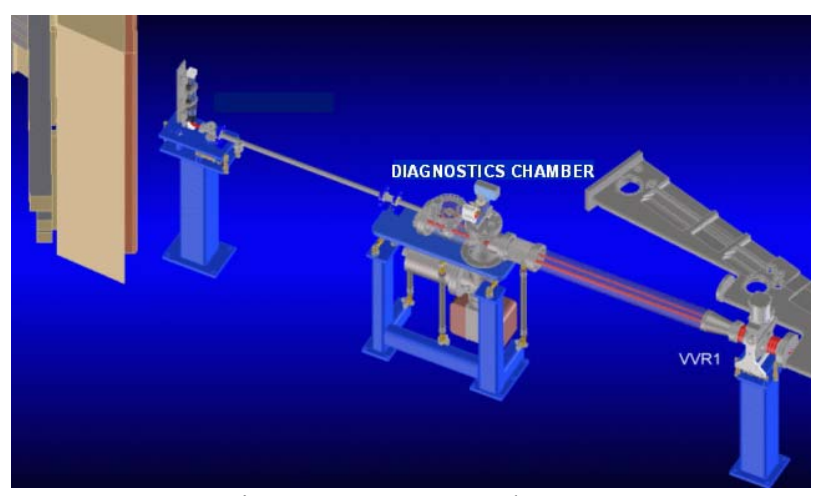

Figure 1: BL 7.2 3D layout.

There is a vacuum chamber situated about $6 \mathrm{~m}$ downstream of the source point that contains the pinhole and the electrodes of the x-ray BPM, see Figure 2.

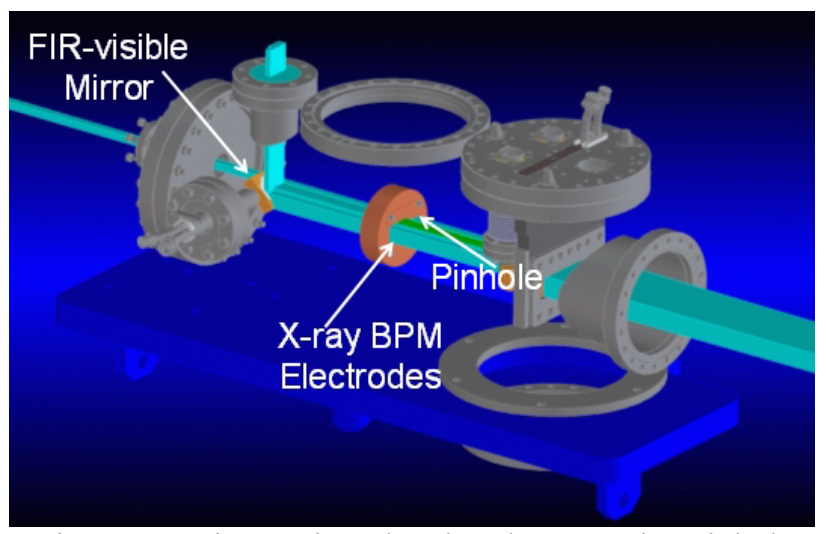

Figure 2: Diagnostics chamber layout. The pinhole array, $\mathrm{x}$-ray BPM, and FIR-visible mirror and vacuum window can all be seen.

In the same chamber and downstream of the electrodes is the 45 degree mirror that sends the visible and the FIR light upwards and out of the chamber. This mirror has 
been machined on top of a copper photon stop and has been coated with aluminium for a better reflection of visible light. A final thin layer of aluminium oxide on the mirror absorbs most of the near-ultraviolet light, improving the safety of the beamline. The synchrotron radiation from the mirror leaves the vacuum chamber through a fused silica window that transmits the visible and also the FIR frequencies. Finally, an optical channel takes the light from inside the ALS shielding outside to an experimental area, where an optical table contains all the instrumentation required for the measurements. The optics on this line are completely based on the use of mirrors for avoiding chromatic aberration. The overall length of the visible photon line from the silica window up to the optical table is about $6 \mathrm{~m}$.

\section{MEASUREMENTS}

\section{Emittance and Momentum Spread}

The ALS diagnostic beamlines take advantage of the different dispersion and beta functions at successive bending magnet sources in our lattice. Measurements of horizontal beamsize at the two source points can be combined with these parameters to find the emittance and momentum spread of the electron beam.

The nominal horizontal beta functions are $0.9 \mathrm{~m}$ for BL 7.2 and $0.35 \mathrm{~m}$ for BL 3.1, and the dispersion values are $7 \mathrm{~cm}$ and $3 \mathrm{~cm}$, respectively. For the typically measured horizontal RMS beam sizes of 123 microns at 7.2 and 56 microns at 3.1 , the natural emittance of the machine is found to be $7.5 \mathrm{~nm}$, and the natural relative momentum spread is $0.1 \%$.
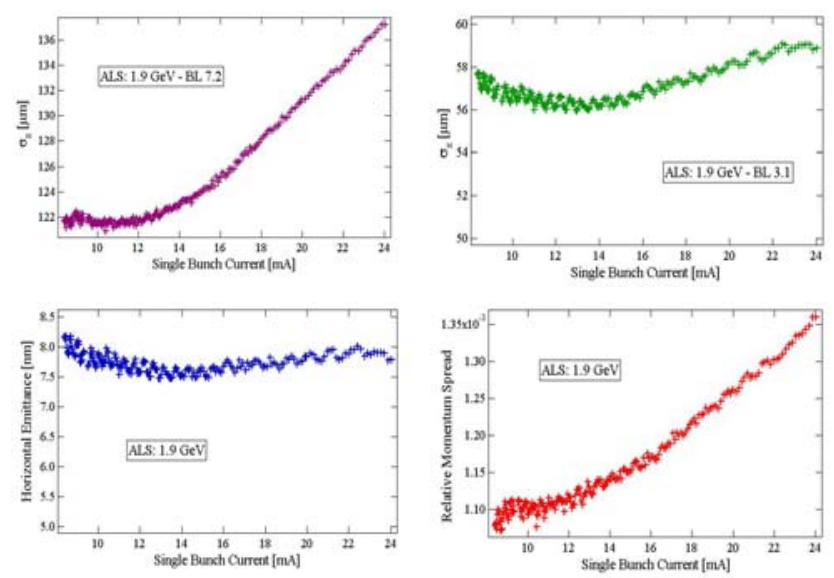

Figure 3: Horizontal beamsizes at BL 7.2 and 3.1 as a function of current per bunch, along with the calculated emittance and momentum spread.

Figure 3 shows an example of emittance and momentum spread measurement versus current per bunch. The horizontal beamsizes at BL7.2 (upper left) and BL3.1 (upper right), are combined to find the natural emittance (lower left) and momentum spread (lower right). Note that the microwave threshold is clearly visible at about 11$12 \mathrm{~mA}$.

The figure also illustrates some problems that we have with BL 3.1. In the low current per bunch region, the measured beamsize shows an anomalous increase with decreasing current. This is due to the poor background subtraction capabilities of our current commercial image analyzer, which break down at low beam currents when the signal to noise ratio in the acquired image worsens. Additionally, we think that the small oscillation of the measured beamsize with changing current on BL 3.1 is an artefact of the fitting algorithm of the analyzer software. To solve these problems, we are replacing the image acquisition and analysis system with one identical to our self-developed system used at BL 7.2.

\section{Visible Light Streak Camera Measurements}

As mentioned, the FIR-visible synchrotron radiation photon line has been designed and constructed using only reflecting optics, rather than any diffractive optics. This choice eliminates chromatic aberrations, and allows the use of the full photon flux in the visible spectrum $(\sim 1 \mathrm{~mW})$, while preserving the resolution. The generous flux that results allows for clean distribution measurements even during single bunch, single turn measurements.

Longitudinal bunch distributions at the ALS are now measured using the visible light port of BL 7.2 and a Hamamatsu C5680 streak camera. Figure 4 shows an example of a single bunch measurement integrated over a large number of turns.
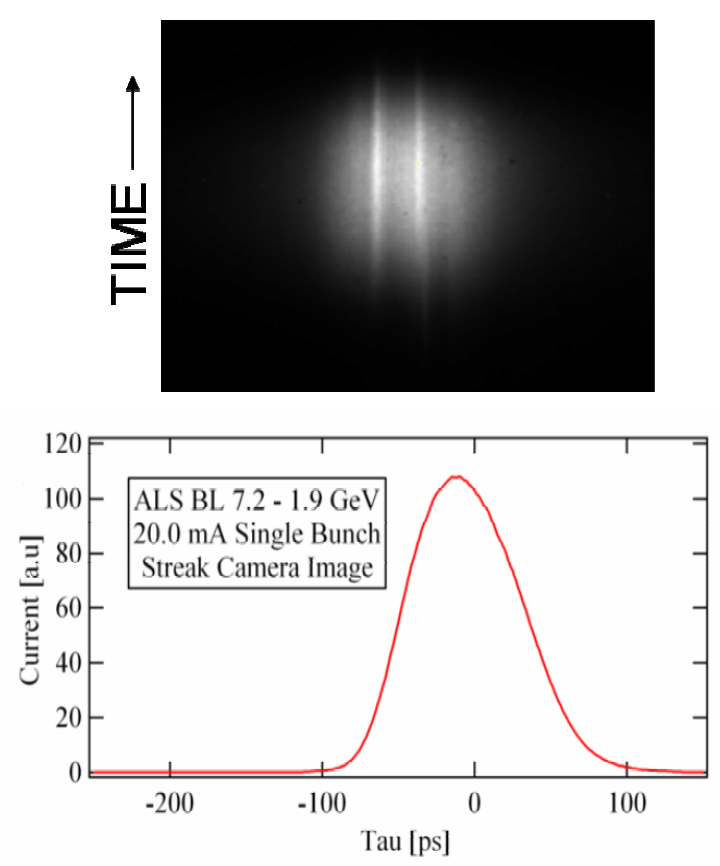

Figure 4: Streak camera measurement at BL 7.2, showing the raw image and longitudinal beam profile. 
Figure 5 shows the case where the streak camera was used in the 'double sweep' mode for single turn single bunch measurements of two bunches, stored in two buckets $328 \mathrm{~ns}$ apart (half the ring circumference), during two-bunch operations at the ALS. The brighter vertical lines in both streak camera images are due to interference in the last part of the optical line; this issue will be addressed in the near future.

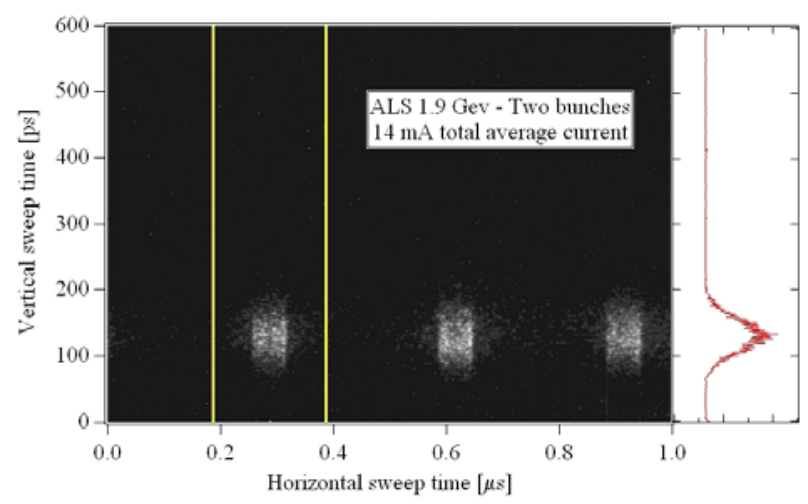

Figure 5: BL 7.2 'double sweep' streak camera measurement of two bunches in the ALS.

\section{Coherent Synchrotron Radiation}

The second branch of BL 7.2 was also designed to transport the FIR synchrotron radiation in the terahertz frequency range, mainly because this band of the spectrum is the one of interest for studying CSR in storage rings.

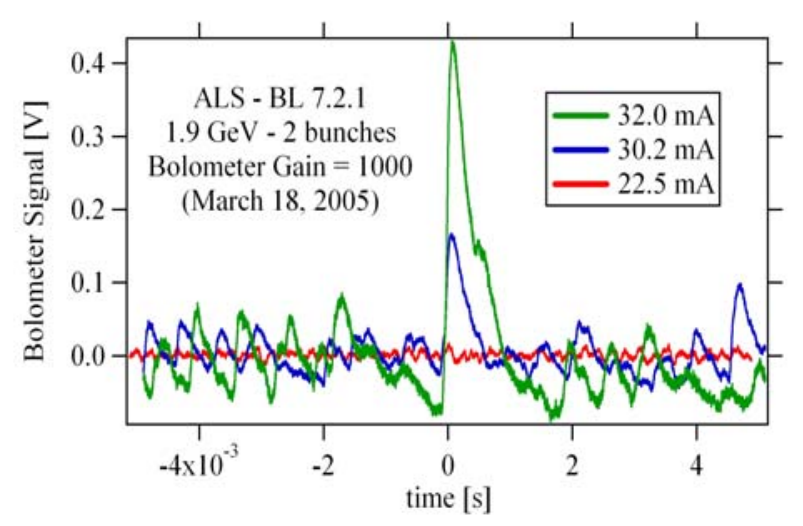

Figure 6: Bolometer signal above and below the threshold for microbunching instability. The coherent synchrotron radiation bursts are clearly visible in the two curves with current above threshold (30.2 and $32.0 \mathrm{~mA}$ ).

Figure 6 shows an example of BL 7.2 measurements of the CSR FIR bursts associated with the microbunching instability (MBI) $[5,6]$. Scope tracks of the signals from the bolometer used for detecting the $\mathrm{THz}$ radiation are shown for different stored current values. The MBI theory predicts that the ALS, in the configuration of the Figure 6 measurements, should have the instability threshold at about $12 \mathrm{~mA}$ per bunch. This is clearly visible in the figure, where the $22.5 \mathrm{~mA}$ curve (11.25 $\mathrm{mA}$ per bunch) does not show any evidence of the strong bursts present in the other two curves with current above threshold. The shape of the burst pulses in the figure is completely defined by the limited bandwidth $(\sim 1 \mathrm{kHz})$ of the detector.

\section{FUTURE PLANS}

The electronics for the X-ray BPM are in place, and the system is ready for commissioning. The main advantage of having a beamline x-ray BPM, in addition to the electron beam BPMs we already have, is that both position and angle information about the bending magnet source point can be simultaneously measured. The output of the BPM may eventually be used to improve our orbit feedback systems.

The Far-IR and visible optical channel of BL 7.2 will be enclosed in a vacuum system with a pressure of about $10^{-3}$ Torr. This will clean up CSR measurements by eliminating water absorption features from the $\mathrm{THz}$ spectra.

\section{AKNOWLEDGEMENTS}

We would like to thank J. Krupnick and D. Robin for their continuous support, the other members of the ALS Accelerator Physics Group for good ideas and advice, the ALS Mechanical and Vacuum Technicians, and the ALS Computer Controls Group.

\section{REFERENCES}

[1] F. Sannibale et al., "A second beam-diagnostic beamline for the advanced light source", PAC 2003, Portland, OR USA, May 12-16, 2003.

[2] F. Sannibale et al., "Commissioning of BL 7.2, the new diagnostic beamline at the ALS", EPAC 2004, Lucerne, Switzerland, July 5-9, 2004.

[3] F. Sannibale, "Experimental Error Analysis of a Possible Measurement of the Emittance and Momentum Spread at the ALS", ALS Note LSAP-301 (2003).

[4] W. B. Peatman and K. Holldack, "Diagnostic front end for BESSY II”, J. Synchrotron Rad. 5, 639 (1998).

[5] S. Heifets and G. Stupakov, Phys. Rev. ST Accel. Beams 5, 054402 (2002).

[6] J.M. Byrd, et al., Phys. Rev. Lett. 89, 224801 (2002). 